\title{
Modelling of the diffusion of carbon dioxide in polyimide matrices by computer simulation*
}

\author{
E. Smit, M.H.V. Mulder, C.A. Smolders, H. Karrenbeld, J van Eerden and D. Feil \\ Department of Chemical Technology, University of Twente, P O Box 217, 7500 AE Enschede (The Netherlands)
}

(Received August 5, 1991, accepted in revised form June 11, 1992)

\begin{abstract}
Computer anded molecular modelling is used to visualize the motion of $\mathrm{CO}_{2}$ gas molecules inside a polyımide polymer matrix The polymers simulated are two 6FDA-bases polyımides, 6FDA-4PDA and 6FDA-440DA These polymers have also been synthesized in our laboratory, and thus the simulated properties could directly be compared with "real-world" data The simulation experiments have been performed using the GROMOS 1 package The polymer boxes were created using the soft-core method, with short (11 segments) chains This results in highly relaxed and totally amorphous polyimide matrices The motion of randomly placed $\mathrm{CO}_{2}$ molecules in the boxes durıng molecular dynamics runs was followed, revealing three types of motion jumping, contınuous- and trapped motion The calculated diffusivities are unrealistic, but possible shortcomings in our model are given
\end{abstract}

Keywords diffusion, gas and vapor permeation, theory, computer modelling, polymer matrix

\section{Introduction}

The permeation and diffusion behavior of gases through polymers cannot be completely described by the macroscopic models normally used. Several models have been proposed to explain the diffusion behavior of small molecules in polymers. These models can be classified as

(1) molecular or matrix models [1-5], or as

(2) free volume models [6-8]

The matrix model relates the diffusivity of a

Correspondence to M H V Mulder, Department of Chemcal Technology, University of Twente, P O Box 217, 7500 AE Enschede, The Netherlands

*Paper presented at the Int Symp on " Progress in Membrane Science and Technology", Enschede, Netherlands, June 25-28, 1991 penetrant molecule to specific polymer-penetrant interactions, and to the change in the matrix that is caused by this interaction. Polymer matrix parameters, such as the flexibility of the polymer chains and matrix structure, are main parameters limiting the rate of diffusion.

In the free volume model the fractional free volume present in the polymer matrix, and the size and shape of the gas molecule are the ratedetermining parameters A major problem is that we are unable to consider the processes on a molecular level However, nowadays computer arded molecular modelling (CAMM) is being used more frequently to obtain that information. CAMM is the most recent and most advanced method avallable at present to simulate a polymer matrix Molecular dynamics 
(MD) simulations can give insight in the diffusion process in such a detall as was previously unattainable $\mathrm{MD}$ may be used to predict the macroscopic properties resulting from interatomic interactions [9] A molecular level description of glassy polymers may someday enable the $a$ prior prediction of structure-properties relationships Current theories are not able to provide us with this ability The technique is available in our department and in cooperation with other researchers (Berendsen and Sok from the Unıversity of Gronıngen, the Netherlands, and Takeuchı from Mitsubishı Kaseı Corp., Yokohama, Japan) an amorphous polymer matrix box has been created, and the diffusion of $\mathrm{CO}_{2}$ through that box was simulated. In this way we were able to simulate the highly complex 6FDA-polyımides which have been synthesized in our group $[10,11]$ This means that computed results could he compared with the actual diffusion measurements

For the MD calculations we used the GROMOS program [12] It makes use of a force-field consisting of a sum of the bonded and nonbonded interactions between the atoms in the system to be studied:

$$
\begin{aligned}
E_{\mathrm{tot}}=E_{\text {bond }}+E_{\text {angle }} & +E_{\text {dihedral }}+E_{\text {improper }} \\
& +E_{\mathrm{VdW}}+E_{\text {electrostatic }}
\end{aligned}
$$

The bonded interactions describe the interactions between covalently linked atoms.

$$
\begin{aligned}
& E_{\text {bond }}=\sum \frac{1}{2} k_{\text {bond }}\left(r-R_{0}\right)^{2} \\
& E_{\text {angle }}=\sum \frac{1}{2} k_{\text {angle }}\left(\Theta-\Theta_{0}\right)^{2} \\
& E_{\text {dihedral }}=\sum k_{\text {dihedral }}[1+\cos (n \Phi-\delta)] \\
& E_{\text {1mproper }}=\sum \frac{1}{2} k_{\text {improper }}\left(\Psi-\Psi_{0}\right)^{2}
\end{aligned}
$$

The non-bonded atoms are taken to exhibit pairwise Van der Waals and electrostatic interactions:

$$
\begin{aligned}
& E_{\mathrm{VdW}}=\sum C_{12}\left(r_{1,2}\right)^{-12}-\sum C_{6}\left(r_{1,2}\right)^{-6} \\
& E_{\text {electrostatic }}=\sum\left[q_{1} q_{2} /\left(4 \pi \epsilon_{0} r_{1,2}\right)\right]
\end{aligned}
$$

The constants $k$ represent the different force constants, $r$ is the interatomic distance $\Theta, \Phi$ and $\Psi$ denote, respectively, the bond-, dihedral- and the improper (dihedral) angle, and $\delta$ is the dihedral phase shift angle The $k$-values are taken from the CHARMm (version 20 ) as defined in the GROMOs forcefield. The value $n$ denotes the multiplicity of the bond

CAMM packages (like GROMOS) use only static charges on atoms, which can be a limitation as will be shown later

\section{Experimental section}

\section{(1) Simulation of the polymer matrix}

In order to study the diffusional jump process in detail, molecular dynamics (MD) calculations were performed inside 6FDA-4PDA and 6FDA-440DA matrices (see Figs. 1 and 2 for the chemical structures) using $\mathrm{CO}_{2}$ as penetrant gas The motion of $\mathrm{CO}_{2}$ in a simulated polyımide box might provide us with some helpful information From the entire system a topology-file is created, incorporating all bonds, angles, the (improper) dihedrals and the nonbonded interaction pairs within the cut-off range The cut-off radil are set at $6 \AA$ during the shrinking of the box, and $85 \AA$ during the equilibrations and simulations The temperature is set at $298 \mathrm{~K}$, and an NPT ensemble is

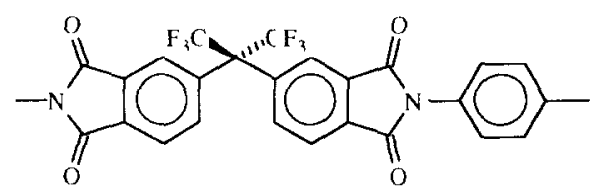

Fig 1 Chemical structure of 6FDA-4PDA

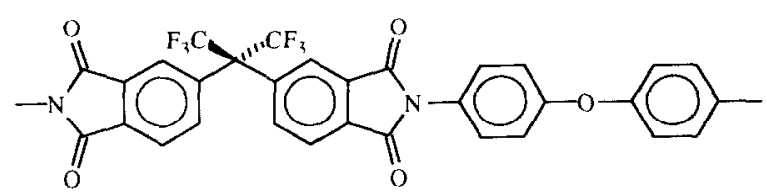

Fig 2 Chemical structure of 6FDA-44ODA 
used The parameters describe through the force-field the energy of the system Integration of Newton's equations of motion over time using all forces gives the time-evolution of the system, in the pico-second (psec, $10^{-12} \mathrm{sec}$ ) range

An amorphous box is created by using the soft-core method, and is used to perform molecular dynamics calculations to simulate $\mathrm{CO}_{2}$ diffusive motion.

The diffusion simulation consists of several parts

(1) Parametrization of the force-field that determınes the dimensions and energetic constraints of the polymer chains

(2) Creation of an amorphous "box" of sufficient size to estımate relevant parameters, and to examine the degree to which the box is amorphous.

(3) Simulation of the diffusion of a small penetrant molecule through this polymer box

\section{(2) Creation of the polymer box}

A commonly used method to create a random box of molecules is based on a self-avoiding random walk [13], but at higher densities (as in our polymers) this Monte-Carlo method often, but not necessarily, fails. We created the polymer matrices by employing the method also used by Sok [9] and Van Gunsteren and Berendsen [12], 1.e the reduction in size of a box with a very tenuous polymer filling to its final, actual density. A problem when simulating bulk polymer matrix formation is the time scale. in reality the formation of e.g a membrane takes several hours, so the time for relaxation of the chains is in the order of $10^{3}-10^{4} \mathrm{sec}$ The time scale for computer simulation is in the order of pico seconds $\left(10^{-12}-10^{-10} \mathrm{sec}\right)$. Hence it will be impossible in a straight-forward box-size reduction experiment to involve all possible chain relaxations This will result in an unrealistic matrix, as indicated by the impossibility to reach the bulk density Thus a mathematical trick has to be used, to take the main obstacle, the direct contact of the polymer chains and the impossibility to relax away from this frictional contact on such a short time scale. This non-relaxable contact would result in a premature freezing of the matrix. The trick that has been applied is to use molecules with a temporarily replacement of the normal Van der Waals (Lennard-Jones 6-12) potential for intermolecular interaction by a so-called soft-core repulsion. We thus allow close contacts between atoms, and the individual chains can even traverse each other the thermal motion can overcome the repulsion.

Hence

$$
\begin{gathered}
E_{\text {soft-core }}=V_{\max }\left[1-\left(r / r_{\min }\right)^{2}\right]^{2} \\
\text { for } r<r_{\min } \\
E_{\text {soft-core }=0} \text { for } r \geqslant r_{\min }
\end{gathered}
$$

where $V_{\max } \approx k T$, 1 e the maximum repulsion energy is in the order of $k T$. This is visualized in Fig. 3

Also the electrostatic potential is neglected during the reduction of the size of the box Since now the premature freezing of the matrix is prevented no complex relaxation processes occur during the matrix formation, and also no minimization during the volume reduction is necessary In this way the simulation process is clearly accelerated The box which has been simulated contains five randomly orientated

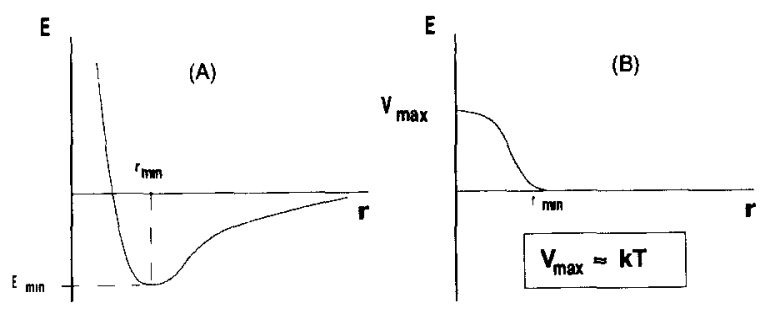

Fig 3 Lennard-Jones 6-12 potential (a) versus soft-core potential (b) 
polymer chains of eleven monomer segments each (also with random foldıng, as far as strain permits), e.g.:

$$
\begin{array}{r}
\text { PDA - 6FDA - PDA - 6FDA - PDA - 6FDA } \\
\text {-PDA - 6FDA - PDA - 6FDA - PDA }
\end{array}
$$

The box has periodic boundary conditions to prevent surface effects, which means that parts that may leave the box on one side re-enter on the opposite side. Also the non-bonded interactions stretch through the box walls At the start of the simulation process the size of the box is such that it has about $1 \%$ of the bulk density of the polymer. The density has been gradually increased up to the experimental value by reducing the size of the box.

After each small reduction step the box is "shaken" by molecular dynamics procedures to achieve a relaxation of the polymer matrix All significant bending-possibilities and rotatıonal degrees of freedom are taken into consideration in the creation of the matrix. To get a realistic matrix simulation a large number $(10,000)$ of reduction steps are necessary Since the box consists of approximately 1200 atoms, and thus many thousands of interactions (over 50,000 , mostly non-bonded), the calculations are very time-consuming

The result of the simulation with a final softcore potential between the chains is not realistic, of course, and after the bulk density (or a somewhat higher value) is reached, the normal Lennard-Jones and electrostatic potentials are again introduced. Subsequently the system is equilibrated normally The equilibration takes 10,000 steps of 0002 psec (the standard time step in our experıments) each This equilibration results in a limited moving apart of the chains, but not to a significant increase of the box volume. We anticipate that the resulting matrix resembles the real polymer state. Both have an intimately intertwined structure, and are well-relaxed The generated systems, consisting of five polymer chains, are cubic simulation boxes with periodic boundary conditions. The box edge after shrinking is about 25 $\AA$ The 6FDA-PDA box has a final density of 1.409 after shrinkıng, and 1.266 after equilıbration. For 6FDA-ODA these values are 1.445 and 1.165 .

In order to check the amorphous character of the boxes (6FDA-polyimides are completely amorphous), a test for parallel ordering of (segments) of the polymer chains was performed. The orientation-correlation function:

$P_{2}=\frac{1}{2}\left[3\left(\cos ^{2} \phi\right)-1\right]$

was used to evaluate possible intermolecular alignments [13]. With only five chains in the box merely checking the overall chain ordering would give only ten angles, resulting in an insufficient statıstıcal significance. Therefore we divided every chain into sub-units (one 6FDA and one 4PDA or 440DA unit, see Fig. 4) In this way 40 angles are considered. The result of the test for the polymer boxes is a $P_{2}$ value of about $0.02 \pm 0.01$. This low value indicates that the boxes are almost completely amorphous: perfect random ordering would yield $P_{2}=0$

As an example a two-dimensional represen-

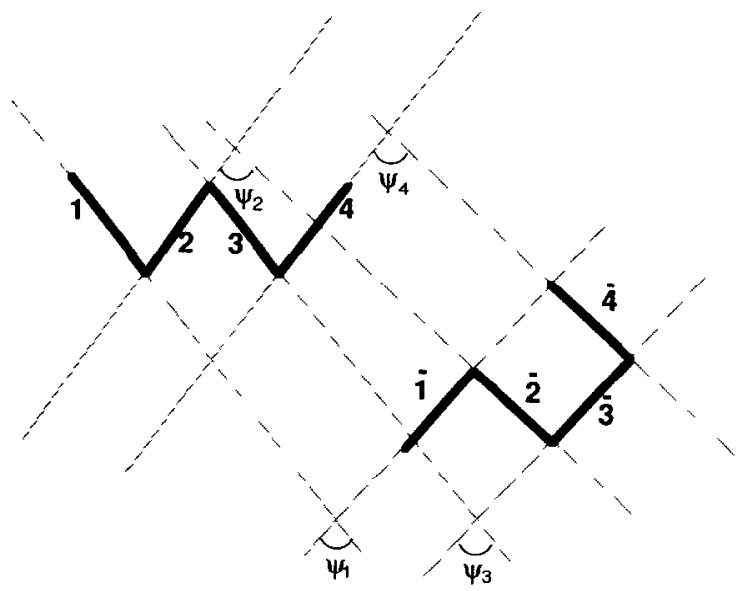

Fig 4 Test for parallel ordering of chain segments in the polymer box 
tations of one of the polymer boxes thus created is given in Fig. 5 (6FDA-44ODA). All atoms are given. Of course the picture displays only a snapshot, since the system is changing in time.
(3) Modelling of the diffusion process by molecular dynamics (MD)

One, respectively ten, $\mathrm{CO}_{2}$ molecules are placed into the boxes of 6FDA-4PDA and 6FDA-440DA at random positions We believe

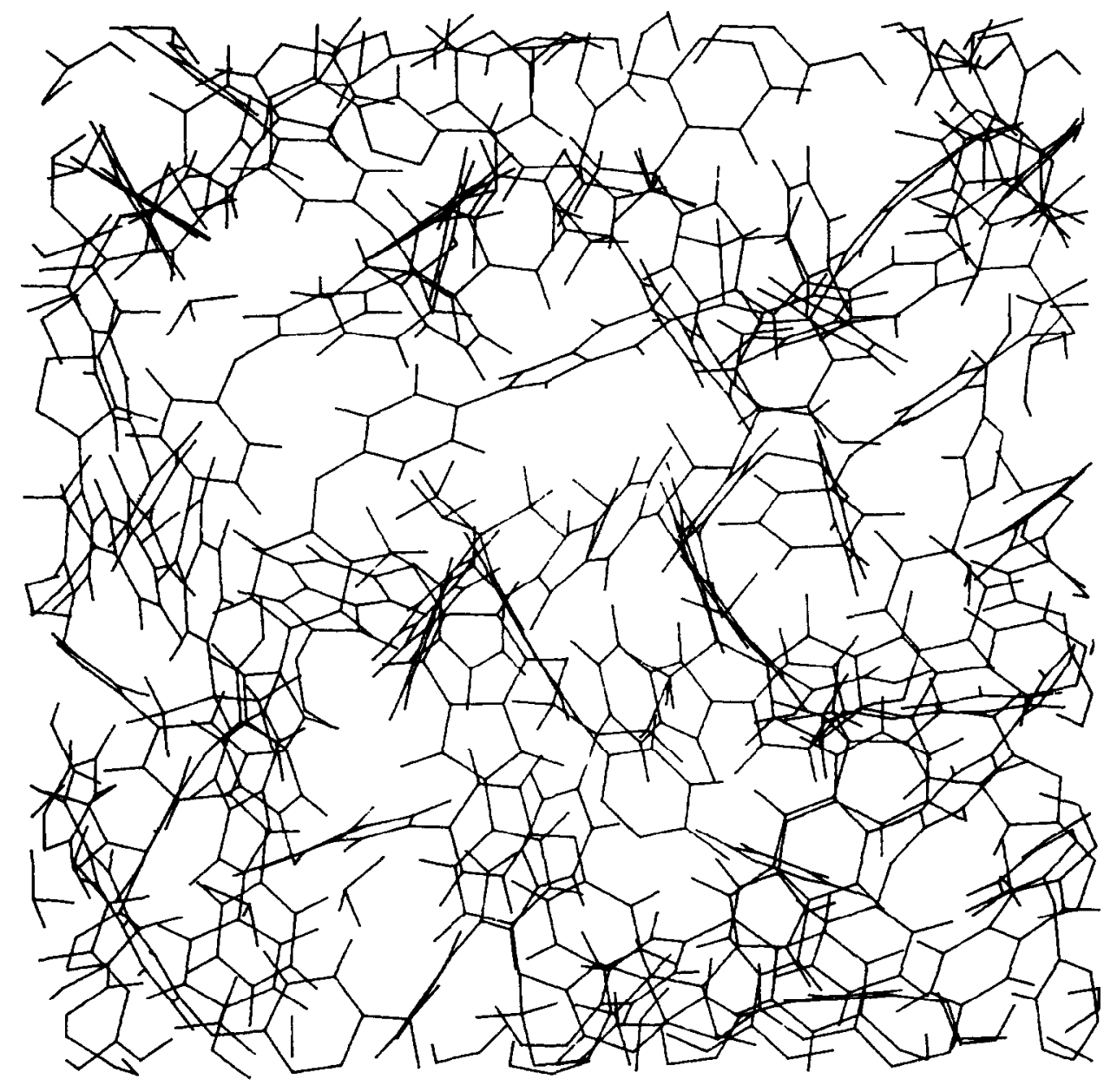

OLY-ODA-6FDA, 1 CHAIN, 11 UNITS, EM, HK

Fig 5 2D representation of the 6FDA-44ODA polymer box 
that at this loading no considerable swelling will occur The $\mathrm{CO}_{2}$ molecule is considered as a solvent molecule by the GROMOS package Furthermore it is taken as a stiff rod, kept nearly straight: 179 . The deviation was necessary due to the program-routine that keeps the molecule from bending. In consecutive MD runs at $298 \mathrm{~K}$ (NPT) the diffusive motion of the penetrant is simulated. All possible interactions and chain motions were taken into account during the simulation of the diffusion process. After placement of the gas molecule (s) the entire system is equilibrated This allows stress buldup by the random and thus possibly llogical placement of the $\mathrm{CO}_{2}$ molecules to relax away Again 10,000 steps of 0.002 psec were used in the equilibration. The density of the boxes increases slightly. for 6FDA-PDA it changes from 1266 to 1268 , for 6FDA-ODA the increase is from 1165 to 1196 This represents the strong gas-matrix interactions

From here on the motion of the gas molecules in consecutive MD-runs is followed. For 6FDA-PDA 100,000, and for 6FDA-ODA 40,000 steps are used In Fig 6 an example of the motion of a Ca molecule inside the matrix is given

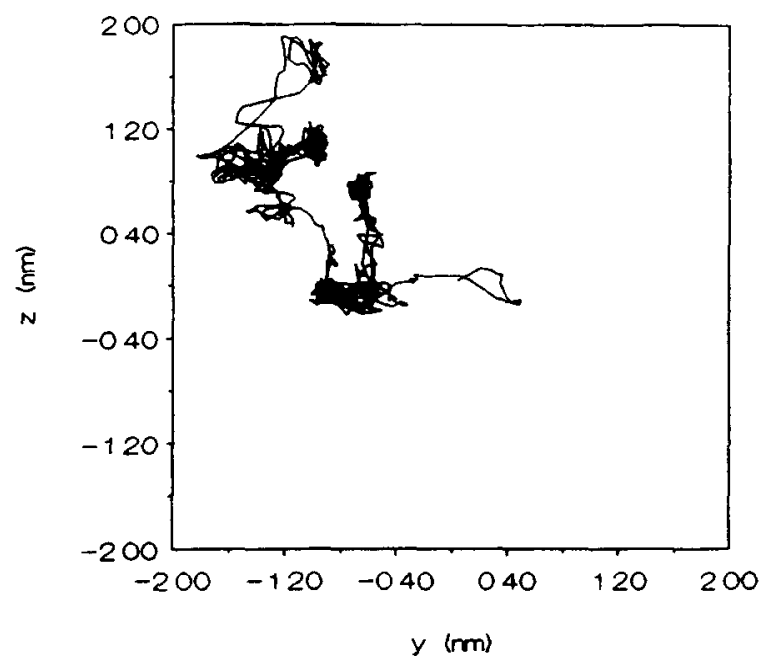

Fig 6 Motion of a $\mathrm{CO}_{2}$ molecule in a $6 \mathrm{FDA}-44 \mathrm{ODA}$ polymer matrıx (a 200 psec run). For clarity only the boundaries of the box are given Here it can already be seen that the gas molecule does not move continuously through the matrix, but discontinuously.

When the displacement from the origin of a $\mathrm{CO}_{2}$ molecule is followed diffusional jumps can be observed In $\mathrm{F}_{1} \mathrm{~g}$ the motion of a $\mathrm{CO}_{2}$ molecule as a function of time is given During the first 100 pse of this particular simulation the gas molecule is "bouncing" at a high frequency inside a hole of about $5 \AA$ Then it moves withın a very short time over a distance of approximately $10 \AA$, a diffusional jump Next it moves inside a confined space again This simulation process is depicted schematically in Fig 8. The tunnel as shown in Fig. 8 is only temporary large enough to let the $\mathrm{CO}_{2}$ molecule pass The poly-

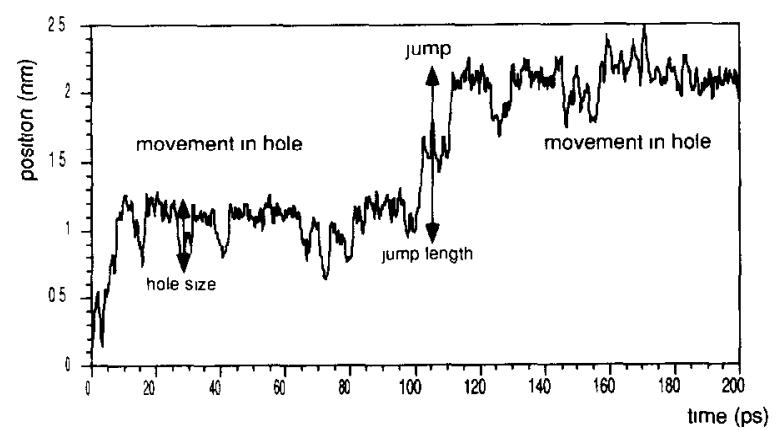

Fig 7 Displacement of a $\mathrm{CO}_{2}$ molecule in a $6 \mathrm{FDA}-4 \mathrm{PDA}$ polymer matrix

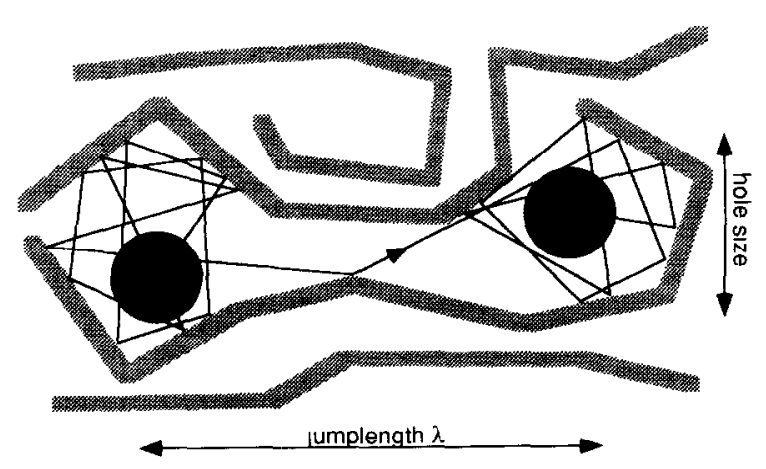

Fig 8 Confined movement and jump motion inside the matrix 

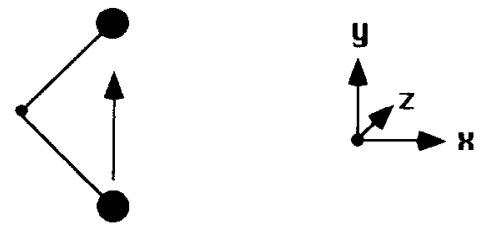

Fig 9 Motion without effective displacement in the $x$ and $z$ direction

mer chains are moving also, but at a much slower rate

To view only the total displacement with regard to the starting point does not yield all detals of the motion, as is exemplified in Fig 9. The total displacement is denoted by $r ; x, y$ and $z$ signify the displacements in these particular
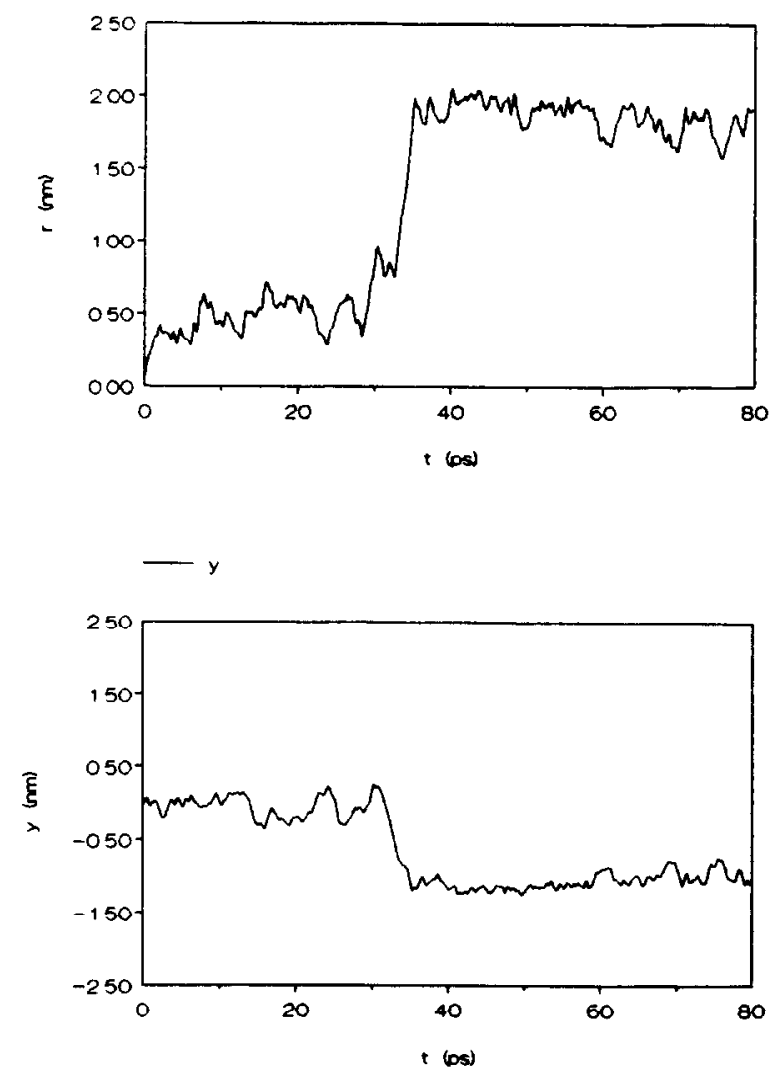

directions Therefore we must consıder the migration in all three dimensions In this way more jumps are revealed The same procedure was followed for 6FDA-440DA The results for one of the $\mathrm{CO}_{2}$ molecules are shown in Fig. 10 (movement from the origin, and in the three directions) In Fig 10 leaps of over $10 \AA$ are visible, indicating true jumps It seems that the jump (s) in 6FDA-44ODA are larger, but it is not possible to give a meanıngful conclusion on only this small number of jumps

When the motions of all gas molecules are investigated in detall, three types of motion become visible Besides the jumping motion the gas molecule can move more or less continuously through the matrix as though it is moving
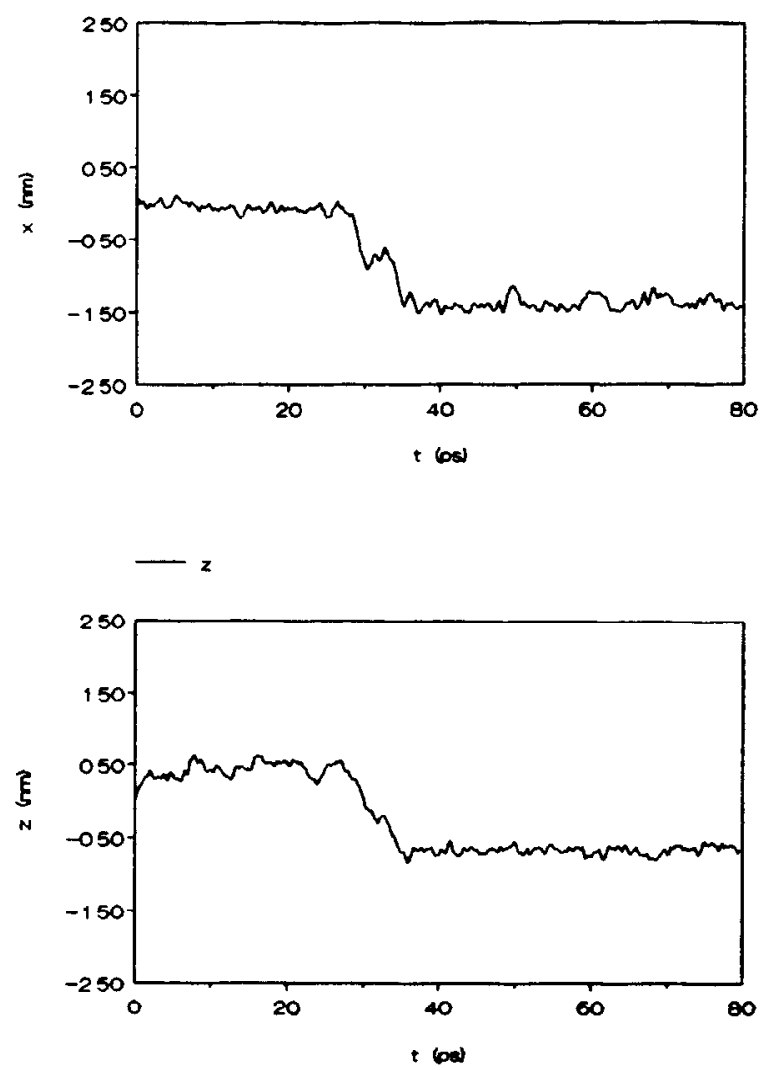

Fig 10 Motions of one of the $\mathrm{CO}_{2}$ molecules in a 6FDA-44ODA polymer matrix 


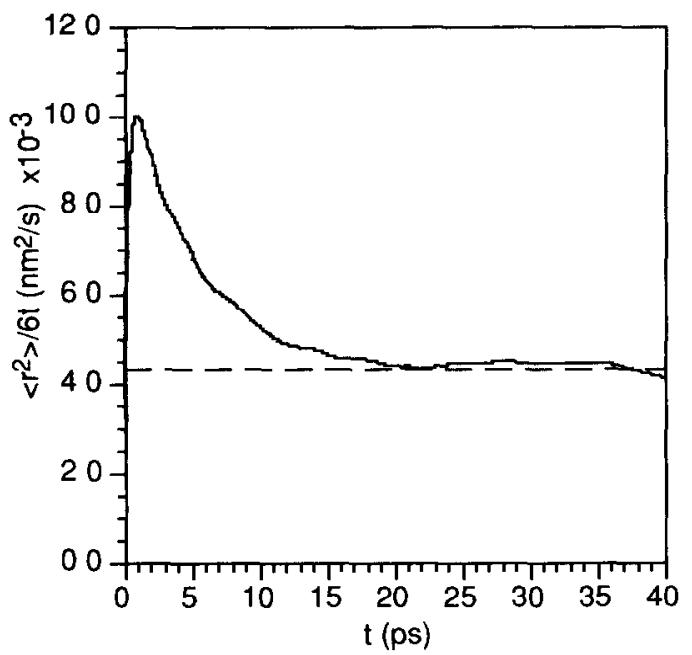

Fig 11 Quadratic displacement from origin versus time in 6FDA-440DA (average of $10 \mathrm{CO}_{2}$ molecules)

inside a large vold or tunnel. The third poss1bility is that the gas molecule is trapped for a long time in a cave. This behavior was also noticed by others [14,15] It encourages our idea that the polymer matrix is the main macroscopic diffusion determining parameter: the chain motions delimit the motion of the gas molecules [10,11]

From the coordinates of the centre of mass of the $\mathrm{CO}_{2}$ molecules the selfdiffusion coefficlent can be calculated using the Einstein relation.

$D=\lim _{t \rightarrow \infty} \frac{\left\langle r^{2}\right\rangle}{6 t}$

where (averaging over all gas molecules and $t_{0}$ )

$\left\langle r^{2}\right\rangle=\left\langle\left|r\left(t_{0}+t\right)-r\left(t_{0}\right)\right|\right\rangle$

For the investigated systems one result is shown graphically in Fig 11 The limiting value represents the selfdiffusion coefficient of $\mathrm{CO}_{2}$ in the polymer box

The resulting diffusion coefficients thus are$D=27 \times 10^{-9} \mathrm{~m}^{2}-\mathrm{sec}$ for $5 \mathrm{FDA}=4 \mathrm{PDA}$ measured: $2.31 \times 10^{-12} \mathrm{~m}^{2}-\mathrm{sec}^{-1}$
$D=43 \times 10^{-9} \mathrm{~m}^{2}-\mathrm{sec}^{-1}$ for 6 FDA-44ODA

measured: $223 \times 10^{-12} \mathrm{~m}^{2}-\mathrm{sec}^{-1}$

The measured data are given in Refs $[10,11]$. It can be seen that the simulated results are considerably too high. Even relatively, between 6FDA-PDA and 6FDA-ODA, the diffusion coefficients are not correct. It should be noted that in our real-world experiments the $\mathrm{CO}_{2}$ concentration is not the same as in our simulation, however

The overshoot at the start of the simulation is caused by the fact that the gas molecule can only move away from its origin in the beginning of the run Only as the simulation proceeds backward motıon becomes also possible

\section{Discussion}

The jump of a gas molecule should be accompanied by a levelling of the barrier imposed by the polymer chains that form the boundary between two holes. This was indeed found by Takeuchı [14]: he notıced a temporary tunnel formation between two holes, and the gas molecule taking advantage of this situation by rapidly moving from one hole to the other. This is in accordance with our model. The motion of the polymer chains is thus the rate limiting factor in the diffusive motion of the gas molecule. Odagakı and Hiwatarı [15] have discovered a similar jump motion behavior in a polymer box, at the glass transition They found a square displacement of molecules in time, and defined the process as follows:

(1) Residence time: during this period the molecule is trapped

(2) Flying time: during this period the molecule makes a large jump

The distributions of the residence time and the flying time have very important physical meaning, especially for the diffusion and the glass transition. No meaningful conclusion about the mean jump distance can be given from the small amount of jumps found so far None- 
theless it seems that the jump length in the 6FDA-44ODA is larger (up to $13 \AA$ ) than in the 6FDA-4PDA box (up to $10 \AA$ ).

The diffusivity values are about three orders of magnitude higher than the experimental values Takeuchı et al. [14,16,17] and others also noticed in rubbery polymers a deviation, although smaller They attributed this to incorrect, albeit small packing density fluctuations caused by the free chain ends or to the crystallinity in the real sample Sefcik et al. $[5,18]$ noticed in ${ }^{13} \mathrm{C}$-NMR rotating frame relaxation experiments a large difference between the effectıve (diffusion) and apparent (NMR) mobility of $\mathrm{CO}_{2}$ in glassy polymers. This seems to indicate that the gas molecules move rapidly, but ineffectively inside holes in the matrix. We assign the difference also to an inaccuracy in the simulated matrix model. The short chains (5 units, 11 segments) have a relatively high amount of end groups $18 \%$ instead of less than $2 \%$ for real polymers. This too high amount of end groups contributes very strongly to the unsatisfactory diffusion coefficient. The mobility of these "loose" ends is much higher than that of the middle of the real chain. This has also been found by others but the effect was not always recognized. Trohalaki et al. [19] measured the free volume as a function of the degree of polymerization (number of segments) of polydimethyl siloxane (PDMS) and polymethylpropyl siloxane (PMPrS, see Table 1).

This table shows clearly that the highly mo-

\section{TABLE 1}

Free volume fraction as function of the degree of polymerization (DP)

\begin{tabular}{lll}
\hline DP & PDMS & PMPrS \\
\hline 10 & 00746 & 00610 \\
15 & 00885 & 00622 \\
20 & 0118 & 00895 \\
25 & 0129 & 0109 \\
\hline
\end{tabular}

blle ends are effective in filling the holes in the matrix: the larger the fraction of free ends, the higher the packing density The chain ends will definitely influence the state of the polymer- the more free ends, the more rubbery (even fluidlike) the polymer will be. The same is true for actual polymers: only a sufficiently high degree of polymerization will allow glass formation. This should have a large effect on the diffusion of gas molecules: the diffusion will be enhanced by the relatively much more mobile chain Takeuchı noticed this chain length effect: if a polymethylene chain was enlarged from 20 units to an infinite number, the free volume increased from 0344 to $0.386(+12 \%)$ [17]. But more interesting is the observation that the relaxation time of internal rotations increase by $50 \%$, and the self-diffusion coefficient of the chain decreased from $022 \times 10^{-6}$ to $8 \times 10^{-8}$ $\mathrm{cm}^{2} / \mathrm{sec}$, a factor of about 30 . This means that the overall chain mobility is significantly reduced when the chain length is increased. We think that especially for glassy polymers this result is very important Since the chain motions limit the gas molecule motion $[10,11]$, an increase in chain mobility will have a large effect on the measured or the computed gas diffusıvity

Another, and maybe the most serious, source of imperfection is the relatively very short simulation time (max. $200 \mathrm{psec}$ ). This will reveil only a small number of jumping processes, and thus might not provide statistically significant data. Probably simulation times in the order of nanoseconds are necessary to obtain useful results Furthermore the CAMM package GROMOs does not take the polarizability of the $\mathrm{CO}^{2}$ molecule and that of the polymer chain into account. This polarizability could result in a much more 'stıcky' matrix, and thus in a strongly reduced gas mobility A third possible flaw could be that our simulated polymer matrix does not represent a realistic state due to the way we created it The switching off of the electrostatic 
interactions during the soft-core shrinking has the consequence that special interchain-interactions that do occur in real are not taken along in a realistic way. We might thus have created a more or less 'alphatic' (no special interactions) matrix. After the shrinking process the electrostatic interactions are again applied, but the very long relaxation times in our system will prevent the formation of realistic interaction possıbilitıes.

\section{Conclusions}

Although CAMM is a new technique, it seems to be a very powerful addition to "classical" methods to elucidate the molecular basis of physico-chemical processes However, the results show some inconsistencies, such as the too high diffusivity, which have to be cleared The calculated diffusion coefficients must be scaled to achieve the same order as the measured values. The question remains: what are these scaling factors, and where do they originate from. Especially in the glassy polymers which we have investigated it was shown that the deviations are very large, a factor of about 1000 . We think that the relatively large amount of end-groups may be the main factor responsible for this anomaly Although we have not yet investrgated this fully, we assume that our system is not really in the glassy state, but at least locally (near the chain ends) in the rubbery state The highly mobile chain ends create large density fluctuations, thereby facilitating the formation of tunnels for the penetrant A determination of the self diffusion coefficient of the chains should give a definite answer.

Also the simulation-time is too short in our experiments. Only the jumps seem to contribute to the effective diffusion coefficient, hence a large number of jumps is necessary in order to use the Einstein equation for the calculation of the diffusion coefficient

Another problem with our system is that mo- delling packages only use static electric charges But $\mathrm{CO}_{2}$ is a non-polar but highly polarizable gas. This may have large interactions with its surroundings, dependent on the selected polymer, an interaction not accounted for in our model. It is plausible that the dipole on $\mathrm{CO}_{2}$ induced by the polar chains reduces its mobility, as long as the matrix is not plasticized by the penetrant. Simulation experiments with nonpolarizable gases such as $\mathrm{N}_{2}$ or $\mathrm{CH}_{4}$ are therefore necessary.

As long as the mentioned problems in the simulation remain unsolved, we think that it is not possible to obtain even semı-quantitative results

\section{Acknowledgement}

The authors wish to thank W Briels (Unıversity of Twente, the Netherlands), R. Sok (University of Groningen, the Netherlands) and H. Takeuchı (Mitsubıshı Kaseı, Japan) for the very helpful and necessary discussions.

\section{References}

1 T -H Kım, W J Koros and G R Husk, Temperature effects on gas permselection properties in hexafluoro aromatıc polyımıdes, J Membrane Scı , 46 (1989) 43

2 D Raucher and MD Sefclk, in TE Whyte, C M Yon and E H Wagener (Eds), ACS Symp Ser No 223, Industrial Gas Separations, American Chemical Society, Washington, DC, 1983, Chap 6, pp

3 H L Frisch, The time lag in diffusion, J Phys Chem , 62 (1957) 93

4 M Salame, Prediction of gas barrier properties of high polymers, Polym Eng Sc1 , 26(22) (1986) 1543

5 MD Sefcck, J Schaefer, F L May, D Raucher, D and S M Dub, Diffusivity of gases and main-chain cooperative motions in plasticized poly (vinyl chlorides), J Polym Sc1, Polym Phys Ed, 21 (1983) 1041

6 W R Vieth, J M Howell and J H Hsieh, Dual sorption theory, J Membrane Sc1, 1 (1976)1 77

7 H Fujita, Diffusion in polymer-diluent systems, Fortschr Hochpolym -Forsch, Bd 3 (1961) 1

8 J S Vrentas, J L Duda and H Ling, Free-volume equations for polymer-penetrant diffusion, $\mathrm{J}$ Membrane Sc1 , 40 (1989) 101 
9 R Sok, personal communications and pre-prints

10 E Smit, Modelling of the diffusion of gases through membranes of novel polyımides, Thesis, Unıversity of Twente, Enschede, The Netherlands, 1991

11 I Blume, E Smit, M Wessling and C A Smolders, Diffusion through rubbery and glassy polymer membranes, Makromol Chem, Macromol Symp, 46 (1991) 237

12 W F Van Gunsteren and H J C Berendsen, Gronıngen Molecular Simulation Library, 1987

13 M Bıshop and J H R Clarke, Brownıan dynamıcs study of the end-to-end distribution function of star and linear polymers in different regimes, $\mathrm{J}$ Chem Phys , 91 (1989) 3721

$14 \mathrm{H}$ Takeuch, A jump motion of small molecules in glassy polymers A molecular dynamics simulation, J Chem Phys, 93 (1990) 2062

15 T Odagakı and Y Hiwatarı, Stochastıc model for the glass transition of simple classical liquids, Phys Rev A, 41 (1990) 929

16 H Takeuchı, R J Roe and J E Mark, Molecular dynamics simulation of diffusion of small molecules in polymers II Effect of free volume distribution, $J$ Chem Phys, 93 (1990) 9042

17 H Takeuchi, Molecular dynamics simulations of diffusion of small molecules in polymers Effect of cham length, J Chem Phys, 93 (1990) 4490

18 M D Sefcrk and J Shaefer, Solid state ${ }^{13}$ C-NMR evldence for gas-polymer interactions in the carbon dioxide-poly (vinyl chloride) system, J Polym Scı, Polym Phys Ed, 1 (1983) 1055

19 S Trohalakı, L C DeBolt, J E Mark and H L Frısch, Estimation of free volume for gaseous penetrants in elastomeric membranes by Monte-Carlo simulations, Macromolecules, 23(1990) 813 